\title{
Ku band transmitarray lens for microwave applications
}

\author{
P. Padilla *, A. Muñoz-Acevedo, J.M. Fernández, M. Sierra-Castañer \\ Universidad Politécnica de Madrid (Technical University of Madrid), Avda. de la Complutense S/N, Madrid 28040, Spain
}

Keywords:

Constrained lens

Transmitarray

Ku band

Microstrip circuit

Patch antenna array

\begin{abstract}
A B S T R A C T
In this document a microstrip constrained lens device for Ku band, for microwave purpose, is presented. This paper offers an overview of artificial lens-type devices and the proposed transmitarray lens is thoroughly studied in terms of design and manufacturing, with architecture discussion and selection, along with the design, manufacturing and validation of all the forming components of the transmitarray (transmission circuits, radiating elements, etc.). Each element is properly characterized and assembled properly in the complete transmitarray prototype. Eventually, radiation pattern measurements as well as gain and directivity values, are provided to show the proper behaviour of the proposed transmitarray lens.
\end{abstract}

\section{Introduction}

It is known that, since actual communication systems are more and more demanding, there is a strict necessity of radiation patterns with hard requirements. Phased array antennas are widely used to overcome these kind of radiation pattern necessities. In this way, there is a growing interest for lens-type or reflector-type structures called transmitarrays and reflectarrays, respectively. These structures replace and, in cases, improve the outcomes of traditional structures, such as reflectors [1] or lenses [2], depending on the structure considered. This paper is focused in transmitarray lens structures and a particular transmitarray for $\mathrm{Ku}$ band is defined, designed, prototyped and measured. The document is organized as follows: Section 2 describes general transmitarray theory. Section 3 offers the complete transmitarray lens device characterization and design, along with some needed considerations. In Section 4, all the constituting elements are designed, prototyped and measured to guarantee their proper functioning. Section 5 describes the assembly and measurements of the manufactured transmitarray prototype and finally, in Section 6, conclusions are drawn.

\section{Transmitarray lens theory}

The basic concepts of artificial lens-type structures can be easily withdrawn: an electromagnetic wave with specific radiation properties (polarization, etc.) and a particular wave front is received in one of the interfaces of the lens, processed in a particular way (change in the radiation pattern, amplification, etc.) inside the lens and finally signal is retransmitted and a new wave front is generated, as sketched in Fig. 1.

Attending to literature, there are different transmitarray models. Some of them [3,4] are relevant due to the proposed multi-beam working scheme: depending on the position of the feeder related to the lens, the main beam direction is going to be different. Regarding the circuitry applied to deal with received signal, lenses are classified into active lenses (if external control signal is used for the inner circuit configuration) [4,5], or passive lenses on the contrary [6-8]. In this work, the attention is focused in the design and manufacture of a complete passive microwave lens with some particular features, as it is described in Section 3. The main value in this device, despite the multi-beam

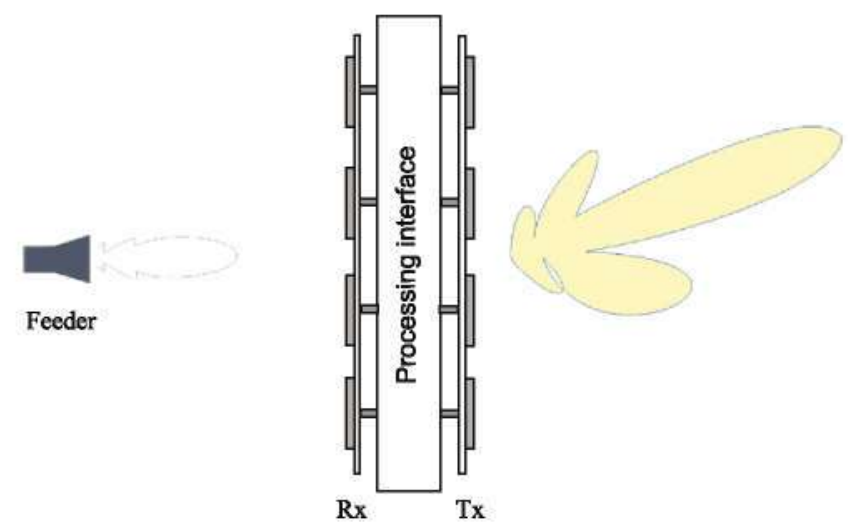

Fig. 1. Scheme of transmitarray lens general functioning. 


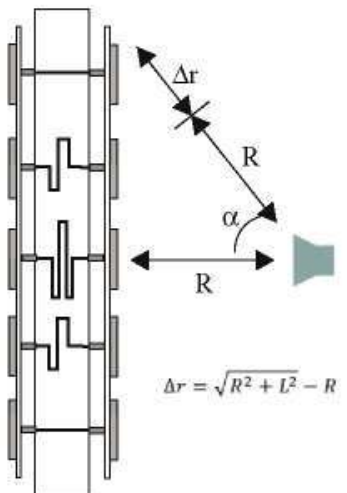

(a)

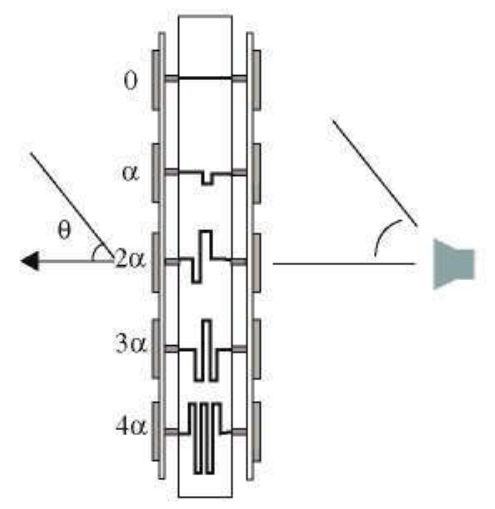

(b)
Fig. 2. Main transmitarray advantages. (a) Phase error correction. (b) Radiation pattern reconfiguration.

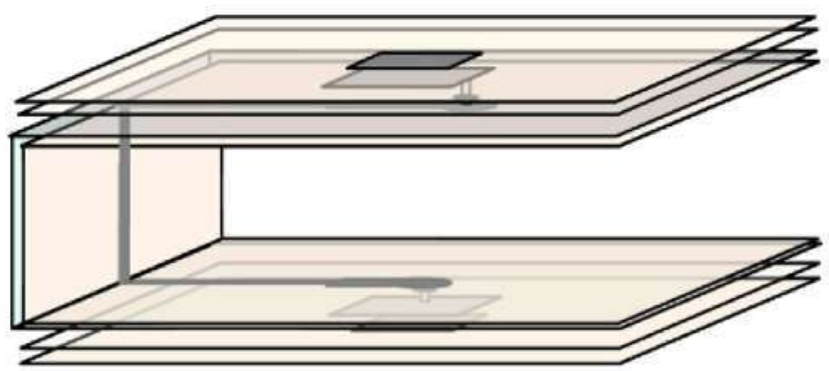

Fig. 3. Scheme of the connection between processing interface and radiating one, with $90^{\circ}$ change in reference plane. functionality, consists of placing it in front of a particular antenna, in order to obtain two main advantages:

- Phase error correction due to spherical-type wave front coming from the feeding antenna.

- New radiation pattern configuration, modifying the phase response of each forming transmitarray cell.

In Fig. 2 these two effects are highlighted.

\section{Transmitarray lens characterization and design}

The transmitarray structure is divided into two principal devices: the radiating interface for reception and transmission and the processing interface, for array phase configuration in each transmitarray cell.

\subsection{Particular transmitarray lens specifications}

The transmitarray design and prototype that is presented in this document satisfies the next requirements:

- Working frequency: $12 \mathrm{GHz}$.

- Band width: $1 \mathrm{GHz}$.

- Matching levels: All the forming elements with a matching level of $-20 \mathrm{~dB}$ or lower.

- Linear polarization. The lens introduces a change in terms of polarization ( $90^{\circ}$ polarization change).

- Special component connection to have space for the processing interface (perpendicular connection between circuits).

- Stacked patches as radiating elements.

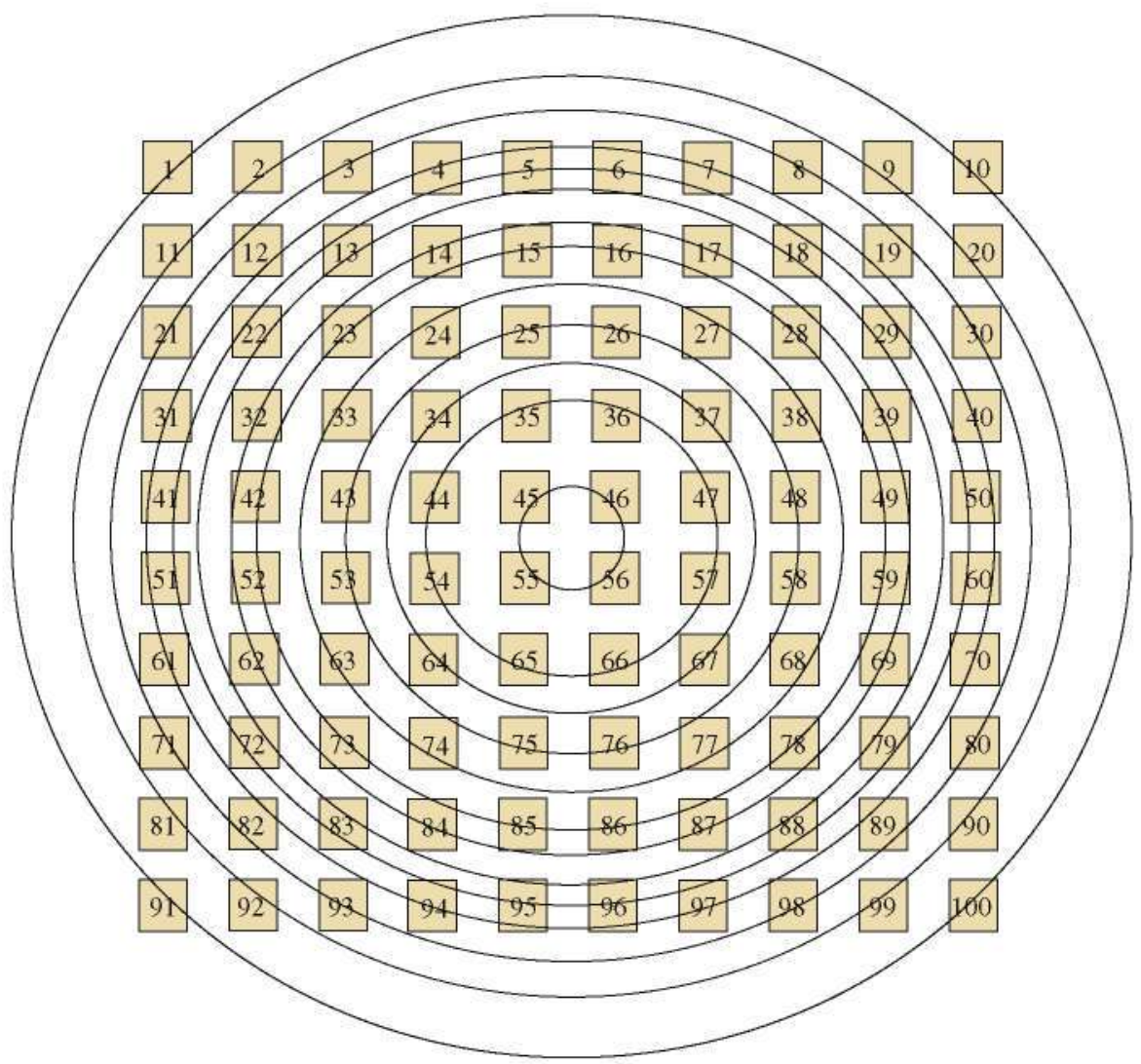

Fig. 4. Ring distribution of the transmitarray cell array for the definition of the pattern configuration. 
- Radiation pattern with $\theta=-10^{\circ}$ of steering direction tilt, for one of the main axes, maintaining the steering direction for the other.

- Feeding antenna: corrugated horn at $12 \mathrm{GHz}$, linearly polarized.

- Number of transmitarray elements: $10 \times 10$ element array.

- Separation between neighbour elements (in terms of vacuum wavelength $\lambda_{0}=0.6$.

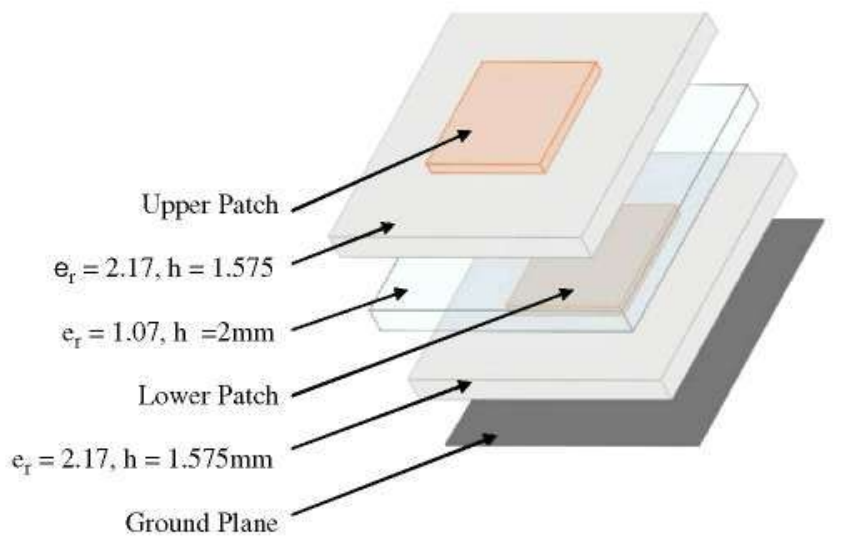

Fig. 5. Model for multilayered patch design, in layer scheme.

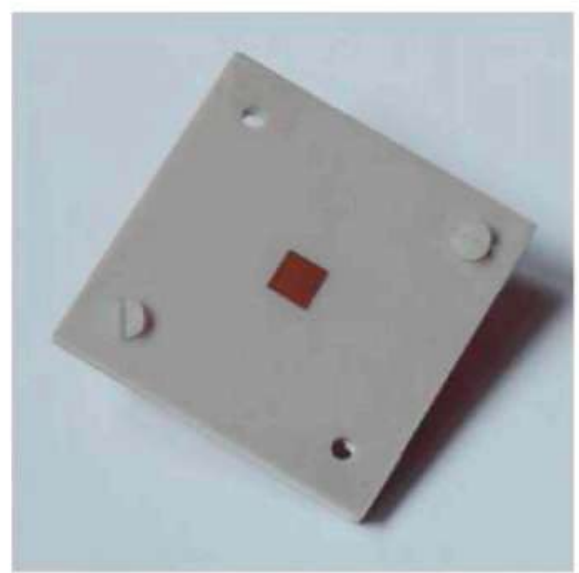

(a)

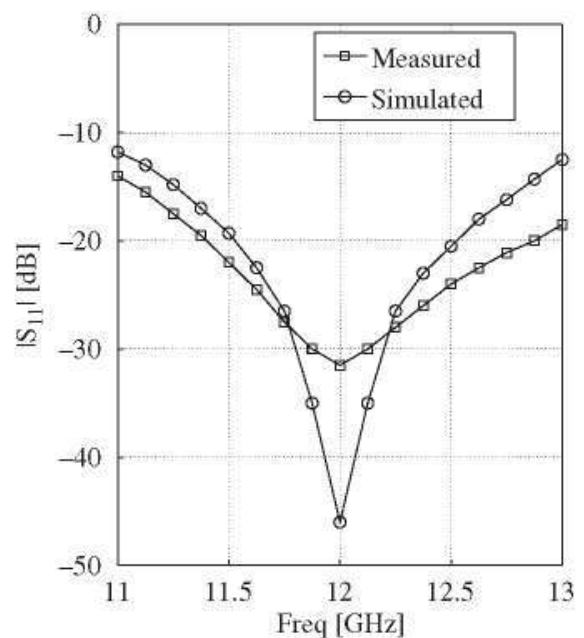

(b)

Fig. 6. Stacked patch cell. (a) Prototype. (b) Simulated and measured results.
- Feeding horn position regarding the lens: $120 \mathrm{~mm}$. This yields $75 \%$ of the radiation pattern, in terms of available power arising in the lens device.

- Ground plane to reflect non-captured power.

\subsection{Geometry applied}

Considering transmitarray structures with planar technology, the most troublesome task is to place the processing interface inside the structure, between both radiating interfaces, being feasible to locate and solder transmission circuits, because of the space available. Both interfaces (processing and radiating ones) which form the transmitarray are designed in planar architecture over ground plane. However, if the connection point between them is discharged of this restriction (planar connection), being possible to introduce $90^{\circ}$ change in the reference plane for each interface by means of perpendicular connection, the space constraint in one of the dimensions is widely reduced, being possible to apply microstrip lines for the processing interface. Fig. 3 exhibits this fact, with the perpendicular connection election between planar interfaces.

In this architecture for the complete structure, all the complexity of connecting the array structures for transmission and for reception by means of microstrip lines is condensed in the connection point between interfaces. This reveals the necessity of

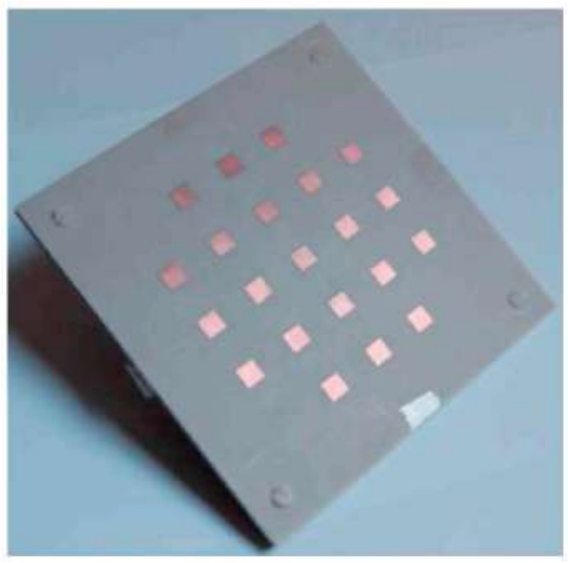

(a)

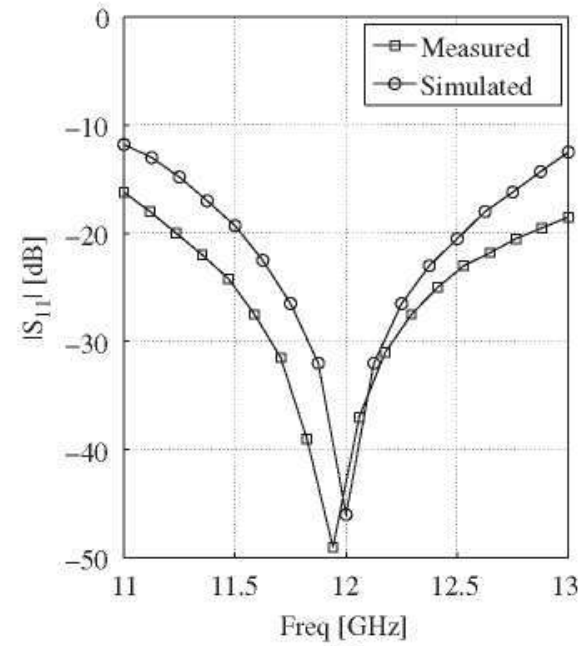

(b)

Fig. 7. Stacked patch cell embedded in array. (a) Prototype. (b) Simulated and measured results. 


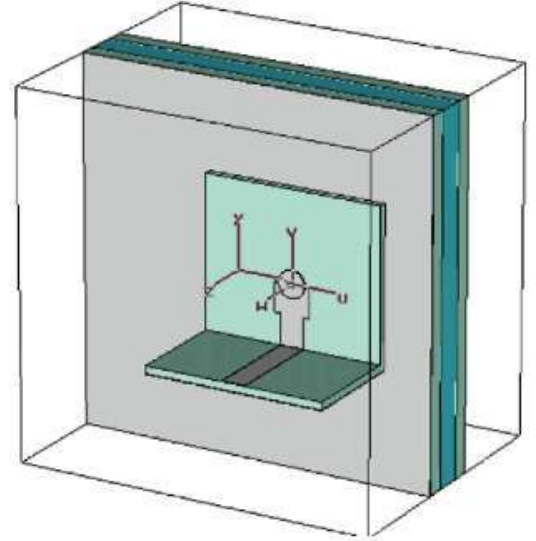

(a)

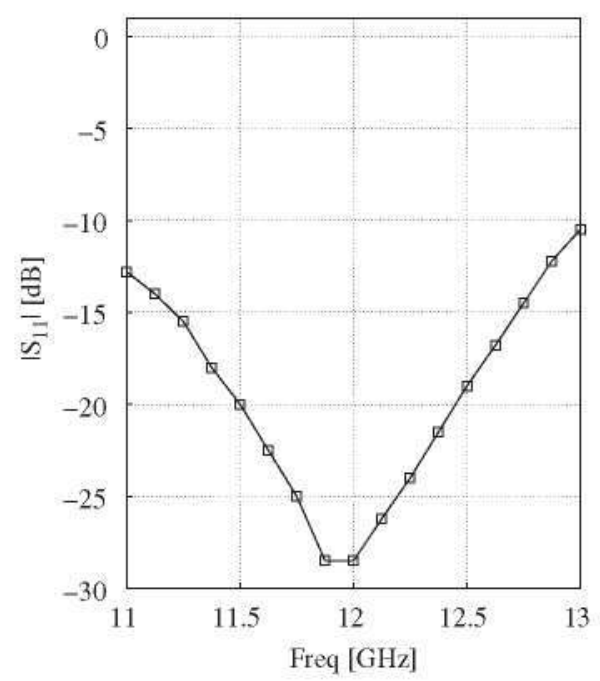

(b)

Fig. 8. Coaxial to microstrip transition and perpendicular connection for simulation. (a) Simulation model. (b) $\left|S_{11}\right|$.

designing a transition between coaxial-type patch connection and microstrip transmission lines, as well as the necessity of designing a perpendicular connection with reference plane change in the microstrip line.

\section{Forming transmitarray elements design and prototyping}

The patch structure is classified depending on the distance of each patch to the feeding horn, depending on the radiation pattern requirements. Patches are classified in concentric rings, as depicted in Fig. 4. According to this, the needed phase error correction together with new phase configuration for pattern modification can be introduced at each cell, in terms of lengths in the transmission lines, in the processing interface.

\subsection{Radiating interface}

The radiating interface is formed by an array of radiating elements. Patches are selected, due to their planar structure, suitable for transmitarray lens devices regarding the selected geometry. In order to increase the bandwidth of the structure, multilayered stacked patch elements over ground plane are applied, rather than simple patch ones, due to their wider

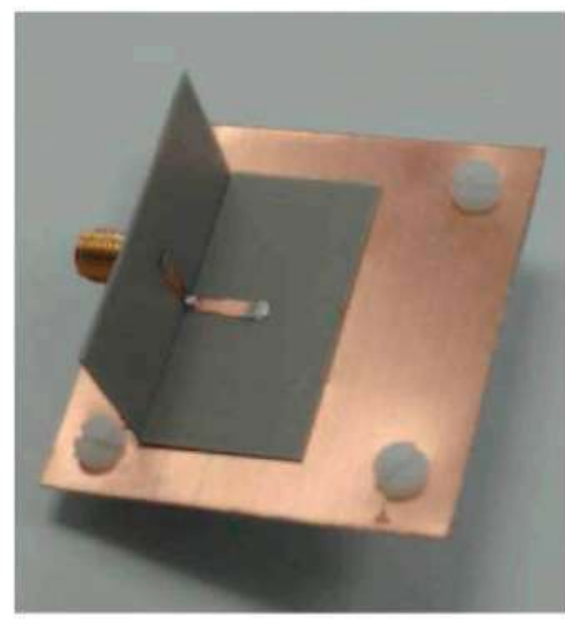

(a)

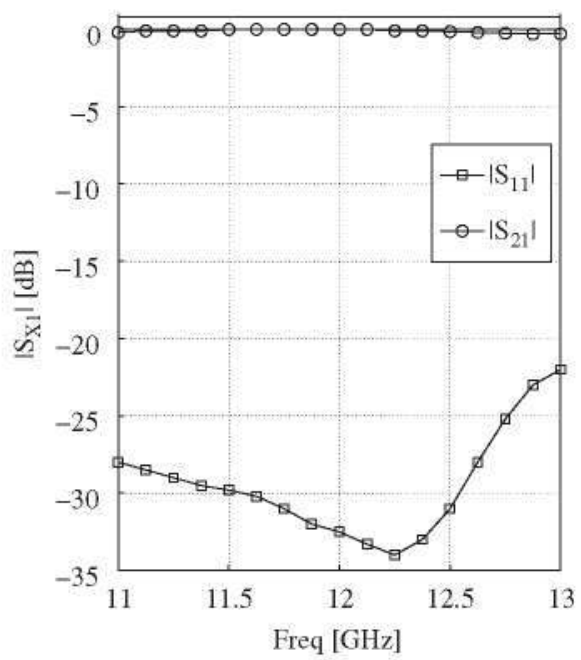

(b)

Fig. 9. Perpendicular connection prototype. (a) Prototype. (b) $\left|S_{X 1}\right|$.

working frequency band. Concerning the design stage, CST microwave studio is applied. Fig. 5 depicts the layer distribution and materials utilized.

In the designing process, one unique stacked patch and stacked patch embedded in array are considered, the last one to include mutual coupling effects with surrounding patches. Figs. 6 and 7 exhibit prototypes along with their design results and prototype results, for a single stacked patch cell and for stacked patch cell embedded in array, respectively.

\subsection{Perpendicular connection and coaxial to microstrip transition}

The perpendicular connection with reference plane change and the one for microstrip to coaxial lines are characterized and designed. Fig. 8 shows design results for these transitions. Fig. 9 shows the prototype and results for these transitions.

\section{Complete transmitarray lens assembly and measurement results}

The final complete assembly to be carried out, for each transmitarray cell, includes the transition device between the 
coaxial-type feeding at each patch and the microstrip lines, and the transition device with perpendicular connection between interfaces, as it is offered in Fig. 10.

Details of the assembly process are depicted in Figs. 11-13. Fig. 14 shows the transmitarray mounted in anechoic chamber for measurement acquisition.

The final prototype is measured in anechoic chamber. Measurement results for the prototype are offered in Fig. 15, along with theoretical results.

These measurements are studied in order to determine measured gain results and expected directivity values. Measurements yield $23.5 \mathrm{dBi}$ in directivity and provide $22.2 \mathrm{dBi}$ mean

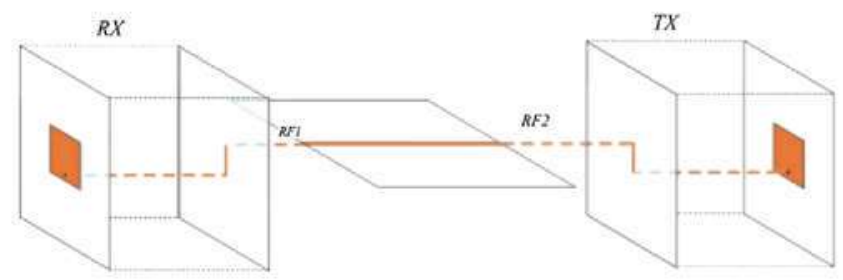

Fig. 10. Assembly model for transmitarray cell.

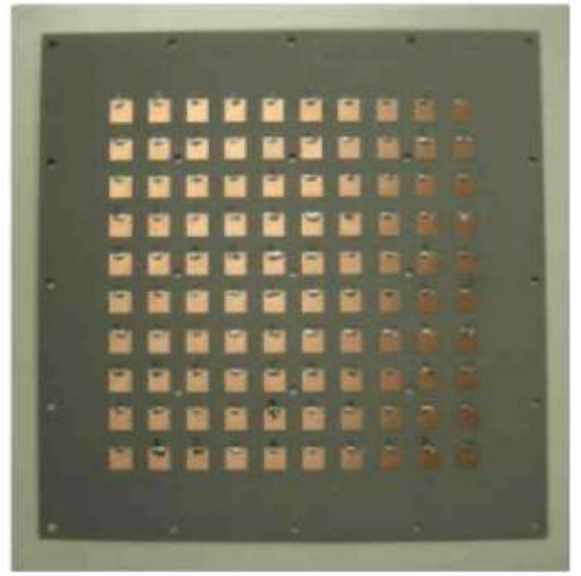

(a)

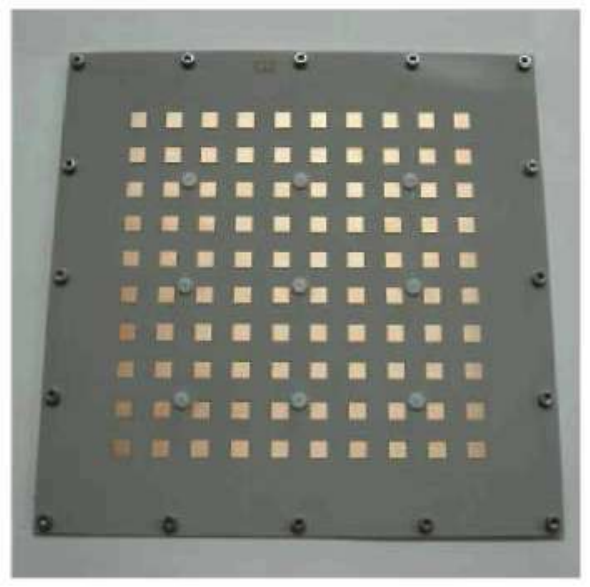

(b)

Fig. 11. Transmitarray radiating interface. (a) Lower array layer. (b) Upper layer.

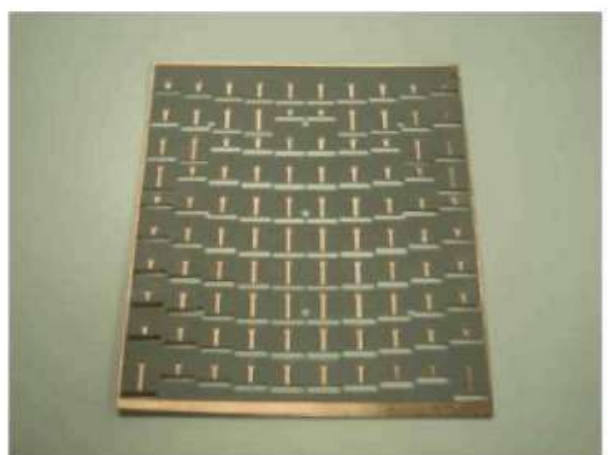

(a)

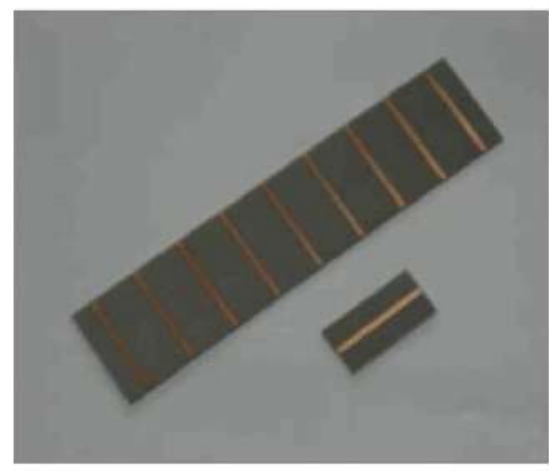

(b)

Fig. 12. Transmitarray processing interface.

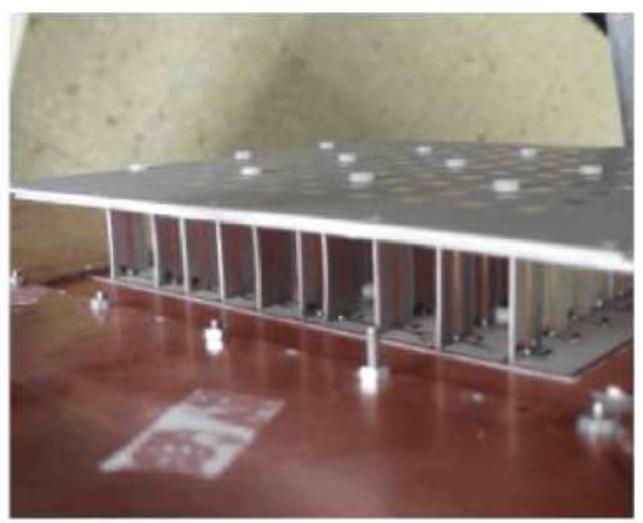

(a)

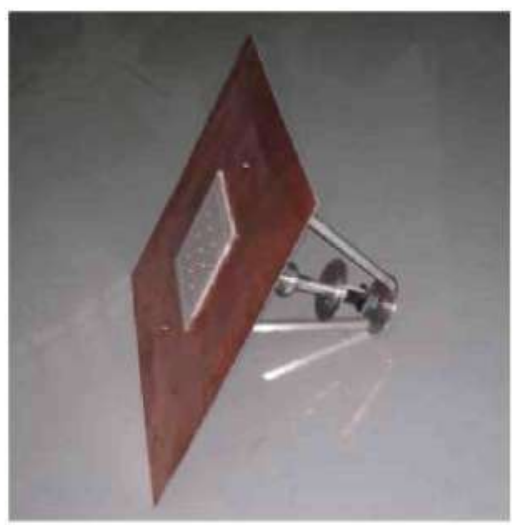

(b)

Fig. 13. Complete assembly (a) Detail. (b) Complete transmitarray. 


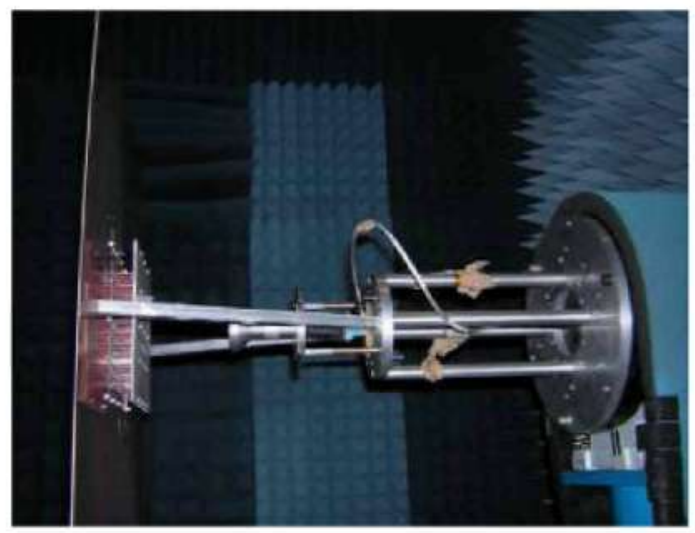

(a)

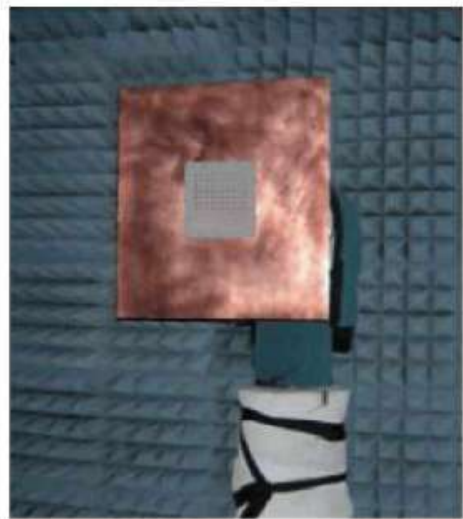

(b)

Fig. 14. Transmitarray in anechoic chamber.

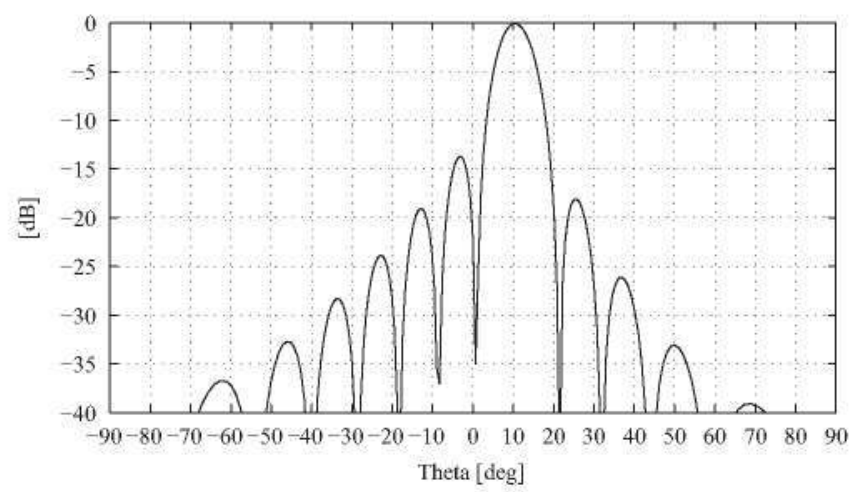

(a)

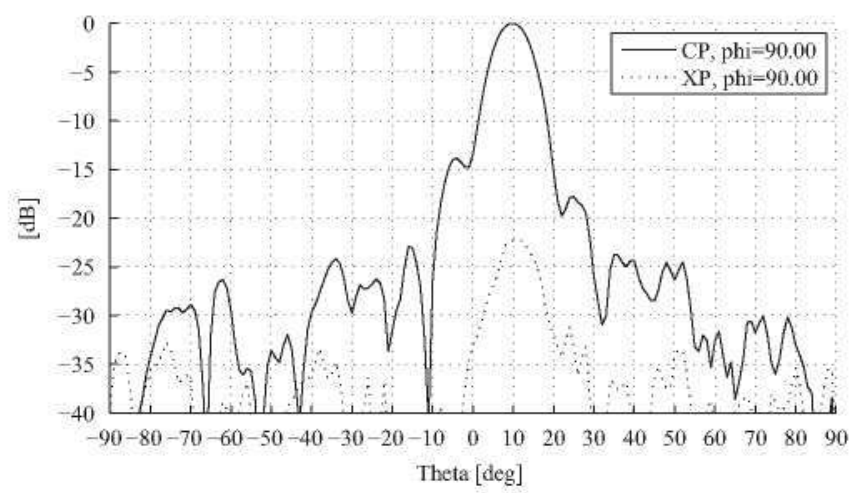

(b)

Fig. 15. Transmitarray measurement results: theoretical radiation pattern (a) versus measurement results (b), for the axis with modified tilt. value in gain at $12 \mathrm{GHz}$. The reduction is due to the accepted horn power (considered that not all the power available in the lens is accepted), and processing interface insertion losses.

\section{Conclusion}

In this document, a complete passive transmitarray lens device has been shown. Some theoretical background and architecture considerations are given and the proper model is chosen, adapted in order to admit the integration of the processing interface inside the complete lens design. A complete transmitarray is designed, manufactured, assembled and measured in anechoic chamber. Deep design and manufacture considerations have been mentioned and a 100 element array device is manufactured. Once the prototype is assembled, measurements in anechoic chamber are obtained in order to achieve the proper behaviour of the transmitarray lens prototype. Main antenna features (gain, directivity, etc.) are extracted and provided.

\section{Acknowledgements}

This research work is been supported by a Spanish Government grant (FPU) and the 'CROCANTE' project (ref: REF:TEC200806736/TEC). The simulations above have been carried out using CST Microwave Studio 6.0 under cooperation agreement between CST and Universidad Politécnica de Madrid (UPM).

\section{References}

[1] Encinar J, Zornoza J. Broadband design of three-layer printed reflectarrays. IEEE TAP 2003;51:1662-4.

[2] Padilla P, Sierra-Castañer M. Design and prototype of a $12 \mathrm{GHz}$ transmit-array. MOTL 2007:49:3020-6.

[3] Garver R. Planar three-dimensional constrained lenses. IEEE TAP 1986;34: 46-50.

[4] Vian J. Popovic Z. Smart lens antenna arrays. IEEE MSD, 2001.

[5] Muñoz-Acevedo A, Padilla P, Sierra-Castañer M. Ku band active transmitarray based on microwave phase shifters. EuCAP, 2009.

[6] Barba M, Carrasco E, Encinar J. Suitable planar transmit-arrays in x-band. EuCAP, 2006.

[7] Costa J, Fernandes C, Godi G, Sauleau R, Le Coq L, Legay H. Compact Ka-band lens antennas for LEO satellites. IEEE TAP 2008;56:1251-8.

[8] Padilla P, Sierra-Castañer M. Design of a $12 \mathrm{GHz}$ transmit-array. IEEE APS, 2007.

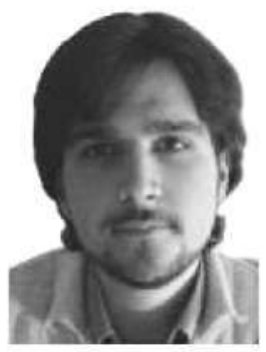

Pablo Padilla was born in Jaén, in 1982. He received the Telecommunication Engineer degree from Technical University of Madrid (UPM), Spain, in 2005 and Ph.D. in 2009. Since September, 2005, he has been with the Radiation Group of the Signal, Systems and Radiocommunications Department of UPM. In 2007, he was with the Laboratory of Electromagnetics and Acoustics at Ecole Polytechnique Fédérale de Lausanne (EPFL), Switzerland, as invited Ph.D. student. In 2009, he carried out a 4 month postdoc at Helsinki University of Technology (TKK). His research interests include antenna design and synthesis and the area of active microwave devices.

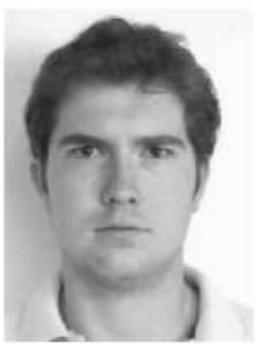

Alfonso Muñoz-Acevedo was born in Toledo, Spain, in 1985. He received the degree of Engineer of Telecommunication in 2008, from the Technical University of Madrid, Madrid, Spain. Since September 2007, he has been with the Radiation Group of the Signal, Systems and Radiocommunications Department of UPM, first as Master Thesis student and since September, 2008, as Ph.D. student. His research interests include antenna design and synthesis and the area of active microwave and millimetre devices. 


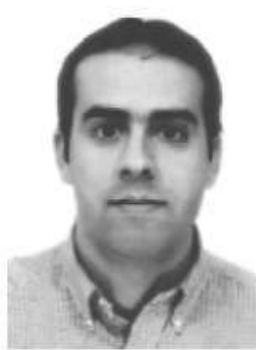

José-Manuel Fernández was born in Lausanne, Switzerland, in 1977. He received the Dipôme d'Ingénieur en Electricité from the Ecole Polytechnique Fédérale de Lausanne (EPFL), Switzerland, in 2003 and the Ph.D. from Technical University of Madrid in 2009. Since 2002, he has been with Grupo de Radiación at Universidad Politécnica de Madrid. His current area of research is in the field of metamaterial structures with emphasis on planar antenna applications. He has been also active in the area of antenna design and synthesis.

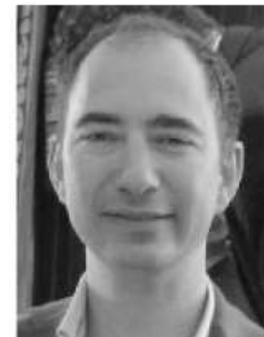

Manuel Sierra-Castañer was born in Zaragoza, Spain, in 1970. He received the Telecommunication Enginee degree in 1994 and the Ph.D. degree in 2000, both from the Technical University of Madrid, Madrid, Spain. Since 1997, he has been in the University "Alfonso X" as Teaching Assistant, and since 1998 at the Technical University of Madrid as research assistant, assistant and associate Professor. His current research interests are in planar antennas and antenna measurement systems. 